\title{
1 Engineered bacterial swarm patterns as spatial records of environmental inputs
}

3 Anjali Doshi ${ }^{1}$, Marian Shaw ${ }^{1}$, Ruxandra Tonea ${ }^{1}$, Soonhee Moon ${ }^{1}$, Anish Doshi ${ }^{2}$, Andrew Laine ${ }^{1}$, 4 Jia $\mathrm{Guo}^{3,4}$, Tal Danino ${ }^{1,5,6+}$

$6 \quad{ }^{1}$ Department of Biomedical Engineering, Columbia University, New York, NY 10027, USA

7 2Department of Electrical Engineering and Computer Sciences, University of California,

8 Berkeley, CA 94720, USA

9 '3ortimer B. Zuckerman Mind Brain Behavior Institute, Columbia University, New York, NY

10 10032, USA

11 4Department of Psychiatry, Columbia University, New York, NY 10032, USA

$12{ }^{5}$ Herbert Irving Comprehensive Cancer Center, Columbia University, New York, NY 10027, USA

13 'Data Science Institute, Columbia University, New York, NY 10027, USA

14

15 †Correspondence should be addressed to T.D. (tal.danino@columbia.edu) 


\section{Abstract}

18 A diverse array of bacteria species naturally self-organize into durable macroscale patterns on

19 solid surfaces via swarming motility - a highly coordinated, rapid movement of bacteria powered

20 by flagella ${ }^{1-5}$. Engineering swarming behaviors is an untapped opportunity to increase the scale 21 and robustness of coordinated synthetic microbial systems. Here we engineer Proteus mirabilis, 22 which natively forms centimeter-scale bullseye patterns on solid agar through swarming, to "write" 23 external inputs into a visible spatial record. Specifically, we engineer tunable expression of 24 swarming-related genes that accordingly modify pattern features, and develop quantitative 25 approaches to decode input conditions. Next, we develop a two-input system that modulates two 26 swarming-related genes simultaneously, and show the resulting patterns can be interpreted using 27 a deep learning classification model. Lastly, we show a growing colony can record dynamic 28 environmental changes, which can be decoded from endpoint images using a segmentation 29 model. This work creates an approach for building a macroscale bacterial recorder and expands 30 the framework for engineering emergent microbial behaviors. 


\section{Main Text}

Swarming behaviors are ubiquitously found in natural systems, ranging from bird flocks to microbial communities, and have inspired creation of artificial systems such as robot swarms ${ }^{6-8}$. A collective movement stemming from individual interactions, swarming can greatly increase a community's scale as well as robustness to noisy individuals and environments. The swarming of many microbial species creates complex emergent patterns at the centimeter-scale on solid surfaces $^{9,10}$. While a long-standing goal of synthetic biology has been to program self-organization in such a fashion, swarming motility has yet to be engineered or used for biotechnological applications ${ }^{11-13}$. Previous approaches have focused on prototypical microbes such as $E$. coli, which forms homogenous colonies, and have engineered swimming and quorum-sensing systems in liquid-agar environments, or utilized external pre-patterning to generate coordinated behavior $^{14-16}$. One promising application of engineering natural swarming is the creation of a durable spatial recording system, using the sensing capabilities of millions of individual bacteria within a swarm to visibly "write" information onto a solid surface. Thus far, synthetic cellular information recording efforts have achieved recording of multiple inputs, cellular lineage, and transient signals, primarily within DNA, but rely on sequencing and other technologies for decoding $^{17-21}$.

We focused on engineering the unique swarming of Proteus mirabilis-a commensal gut bacterium also commonly found in soil and water, which produces a bullseye pattern on solid agar defined by concentric rings of high bacteria density that are visible to the naked eye ${ }^{22}$ (Fig. 1a). The inherent clock-like timing and internal consistency of its ring formation naturally suggest application as a recording system, similar to the way a growing tree records information in the rings in its trunk ${ }^{23}$. Although the ability of $P$. mirabilis to produce rings has been known for over 100 years, it has not been developed as a synthetic biology platform, and quantification of its macroscale patterns has been limited ${ }^{24}$. Beyond the large-scale features of $P$. mirabilis that enable simple decoding visually, applying methods of deep learning and image segmentation can further decode multiple external inputs and dynamic conditions from more complex pattern features.

62 The bullseye pattern of $P$. mirabilis is created from a sequence of phases starting with initial colony 63 growth (lag), followed by oscillatory cycles of synchronized colony expansion (swarming), and 64 stationary periods of cell division (consolidation). The synchronicity of its swarming is achieved 
65 by complex coordination of cell elongation, secretion of surfactant to aid movement, intercellular communication, and alignment of swarmer cells into rafts by intercellular bundling of overexpressed flagella ${ }^{25,26}$. While investigation of the mechanisms governing these behaviors is ongoing, studies have identified an array of genes upregulated during consolidation phases, including those responsible for synthesis of flagella, metabolism, and cell division, and during swarming phases, such as the master regulator $f\left(h D C^{26-30}\right.$. These works have shown that modification of expression of these genes and choice of growth conditions can lead to different variations of the ring pattern ${ }^{31,32}$. We therefore chose the strain PM7002, with baseline conditions that would create a pattern with distinct ring boundaries, such that modifications to the pattern would be easily visible and quantifiable (Fig. S1, S2). After establishing these conditions, we expressed swarming-related genes to controllably modify specific colony pattern features, which could be subsequently analyzed and decoded to report on conditions during colony growth (Fig. 1a).

To initially demonstrate swarm pattern modulation, we engineered $P$. mirabilis with a high-copy plasmid carrying an isopropyl ß-D-1-thiogalactopyranoside (IPTG) inducible promoter, pLac, expressing cheW, a chemotaxis-related gene upregulated in the swarming process (Fig. S12) ${ }^{29}$, 33, 34. In E. coli chemotaxis, CheW is a membrane-bound coupling protein part of a signaling complex in which it bridges the kinase CheA to chemoreceptors, allowing phosphotransfer to CheY and CheB, where CheY is involved in control of flagellar motor rotation ${ }^{35}$. Although the exact role of cheW in swarming is not fully known, a cheW mutant of $P$. mirabilis was previously found to be unable to swarm ${ }^{33}$. Here, inducing constitutive cheW expression with increasing concentrations of IPTG in the agar generated colonies of decreasing ring width and size at 24 hours, compared to a control gfp-expressing strain which showed no change in pattern in response to IPTG (Fig. 1b). To quantify the patterns, we examined the radially averaged pixel intensity as a proxy for colony density in each of the conditions, where high pixel intensity (light colors) represents lower density (Fig. 1c, Fig. S3). All colonies had a characteristically dense boundary around the central inoculum, seen as a dip in the intensity plot around $0.25 \mathrm{~cm}$ from the

93 center of the colony ( $x=0$ in the plot), and showed periodic changes in density across the colony.

94 As expected, the radially averaged intensity profiles showed peaks of intensity corresponding to 95 the periodic ring boundaries. With increasing cheW expression, the profiles showed greater 96 density near the inoculum and at the ring boundaries, which can be seen as lighter areas on 97 heatmaps of radially averaged intensities (Fig. 1d). We constructed a small dataset and 98 measured colony radius manually using image processing tools, and ring widths using a custom 
algorithm (Methods) (Fig. 1e). The colony radius and ring width correlated well with IPTG concentrations $\left(R^{2}=0.90\right.$ for each). To more accurately decode input conditions from the pattern, we fit a multinomial regression model on these measurements and found that the model correctly predicted each colony's input IPTG from the combination of its radius and ring width in all cases (Fig. 1f). We thus reasoned that colony features could potentially encode information about external inputs received by the bacteria, and feature measurements could subsequently be used to decode the information.

\section{Manipulation of multiple swarming-pathway genes}

Given the variety of features observed in $P$. mirabilis patterns in the literature beyond the ring widths and overall colony radii, we explored the potential for multiplexed information encoding. Here we sought to identify additional genes which could distinctly modulate colony pattern features (Fig. 2a). We chose genes previously implicated in a range of points in the swarm process, including $u m o D$, which controls the master regulator of swarming, flhDC; the signaling factors fliA and flgM, which are involved in flagellar gene transcription; and Irp, which affects general cellular processes in response to leucine presence ${ }^{29,36-40}$. Induced expression of these genes via IPTG generated a variety of patterns, ranging from dense ruffled textures, to "spikes", to indistinct ring boundaries (Fig. 2b). Scanning a range of IPTG concentrations showed graded changes in patterns (Fig. S4). For example, with minimal IPTG-induced expression, the Irp strain formed spikes in the inner colony rings, and at maximal induction each ring boundary was spiky. Increased expression of flgM caused colony radius at 24 hours to shrink, while fliA caused the formation of more visible dots or "microcolonies" just within the boundaries of each ring. As umoD expression increased, colonies became more symmetric, and ring boundaries and the inoculum edge became fainter. Taken together, these various qualitative characteristics suggested that induced expression of certain swarm genes could indeed affect several pattern features which could be measured and quantified.

We next examined the radially averaged profiles of each pattern, which revealed distinct characteristics for each strain (Fig. 2c). For example, overall colony density was higher with induced cheW expression than with $u m o D$. The spikes visible in the Irp pattern, which caused ring

129 boundaries to spread over greater widths, reduced the sharpness of the ring boundaries in the 130 radially averaged profiles. Given the repeating nature of features in the patterns, we also explored 131 visualization of the Fourier spectra of the polar transforms of the images, which highlight the 132 presence of frequency information, to see if the spectra varied between strains (Fig. 2d). The 
133 periodic features in the patterns resulted in high visible intensities in certain regions of the Fourier

134 transform images. For example, the $u m o D$ strain displayed a higher magnitude in the outer 135 regions of the transformed images, representing high-frequency (i.e., short distances between 136 repeated features) information, while the other strains showed greater magnitude at the central 137 regions, which represents lower-frequency features. In summary, we saw that the visible 138 differences in patterns between the engineered strains were reflected in, and thus could be 139 analyzed from, quantitative representations of the images.

We next sought to identify features of each strain's pattern which could allow for determination of the input IPTG concentration. We generated a dataset of images for each strain grown at a range of inducer concentrations and measured a range of features for each (Fig. S5a). The low frequencies of the Fourier spectra were found to increase with IPTG induction for the flgM and cheW strains, reflecting the visual observation of thinner, fewer rings of increased density at higher IPTG. (Fig. 2e). A second measure, the local coefficient of variation (CV), increased with increasing IPTG for the Irp strain, which could be observed visually in the spiked rings (Fig. 2f). Finally, the distinctness of the inoculum border, measured by the change in intensity over the border, decreased with increasing IPTG for the umoD strain, particularly from 0.1 to $1 \mathrm{mM}$ IPTG.

(Fig. 2g). These measurements showed that induced expression of these genes could quantifiably affect the pattern in response to changes in IPTG.

As an approach for decoding information from the patterns, we explored fitting regression models on these measurements. The samples were binned into three classes (0-0.09, 0.1-0.9, and 1-10 mM IPTG), and then each feature individually, and all possible combinations of the measured features, were used to fit multinomial regression models, to identify which combination would best decode a given strain's pattern. The performance of such models can be evaluated using a multiclass area under the receiver-operating curve (AUC) metric, where the more accurate a model is for predicting true positives compared to false positives for each class, the closer the AUC will be to 1. The AUC of each strain's fitted model was evaluated on the input data (Fig. $\mathbf{2 h}, \mathbf{S 5 b}$ ). For each strain, the combination of parameters which gave the highest AUC varied, confirming that each strain was encoding information in a characteristic combination of pattern features. The best

163 models for the experimental strains with cheW, fliA, Irp generally had AUC>0.9, showing that the 164 models were well able to differentiate true positives in each IPTG class from false positives. The 165 AUCs were 0.6 for the gfp control strain, just slightly above a random classifier (AUC=0.5), 166 suggesting that pattern parameters were not strongly affected by increasing IPTG for control 
strains. The confusion matrices showed that the fitted models correctly classified a majority of the plates for each strain (Fig. S5c). Thus, information about the environment encoded within the engineered strains' patterns can be decoded using combinations of relevant pattern features.

\section{Dynamics of engineered $P$. mirabilis strains}

$172 P$. mirabilis swarming creates patterns not only in space, but also in time; this temporal regularity 173 suggests the possibility of encoding information in both the endpoint patterns and their dynamic 174 growth phases. We aimed to gain an understanding of the dynamics of the engineered strains by 175 time-lapse imaging of colony growth (Fig. 3a). In order to capture high-resolution images of 176 swarming, we developed a time-lapse setup using a commercial flatbed scanner. For each strain, 177 a time-lapse was captured with maximal IPTG concentration at $25^{\circ} \mathrm{C}$; images were taken every 17810 minutes over the course of the time-lapse (Fig. 3a, Movie S1). The individual images were then radially averaged and full time-lapses were visualized via heatmaps (Fig. 3b). Using a custom semi-automated algorithm (see Methods), we identified the location of the colony front at each timepoint and obtained trajectories with high spatiotemporal resolution (Fig. 3c). The colony growth trajectories showed that each of the engineered strains maintained the classic alternation in phases, but with changes in aspects such as initial lag time and length of the phases compared to the control gfp strain. We then measured the mean length of time of each phase from each of these trajectories (Fig. 3d), which, together with distance swarmed during each swarm phase, enabled the calculation of swarm speed (Fig. 3e).

To explore whether certain dynamic parameters would show a trend with increasing IPTG for each strain, we generated individual time-lapses of each strain grown at a range of IPTG concentrations (Figs. S6-7). When comparing uninduced to induced conditions, we observed distinct measurements for each strain such as the lag time for $u m o D$, the length of the middle 192 consolidation phases for cheW, and the time for the colony to cover the plate for fliA (Fig. 3f). 193 More complex dynamic parameters also encoded information; for example, the asymmetry of the 194 colony front during swarming phases increased with IPTG for the Irp strain (Fig. $\mathbf{3 h}$ ). These results 195 suggest that dynamic parameters can also be used to encode and decode information from these 196 spatiotemporal patterns, and that in the future strains can be chosen for a given application 197 depending on the desired time scale of recording. 


\section{Multiplexed recording using a dual-input strain}

202 In order to build a strain which could provide information about multiple inputs simultaneously, we

203 induced a second swarming-related gene with the pBAD operon and promoter, transcribed in the

204 presence of arabinose (Fig. S9). Since swarming-related genes have interdependent effects, we

205 sought to try two genes which robustly changed distinct pattern features on their own. We thus

206 built a combination strain with cheW expression induced by the pLac promoter, and umoD

207 expression induced by pBAD promoter (Fig. 4a, Fig. S9). Initial characterization of this strain

208 demonstrated that its swarm patterns indeed distinctly reflected the presence or absence of each

209 input (Fig. 4b). Representative radially-averaged profiles were visualized as heatmaps for

210 comparison (Fig. 4c). The plates imaged followed a characteristic pattern at most of the

211 conditions. Increasing IPTG from 0 to $1 \mathrm{mM}$, inducing cheW expression, resulted in a visible

212 decrease in 24-hour colony radius, ring width, and colony symmetry, as seen previously in the

213 single input strain. Meanwhile, increasing arabinose from 0 to $0.1 \%$ resulted in a highly symmetric

214 pattern with initially semi-distinct, narrow rings giving way to the indistinct wide rings more

215 characteristic of the single-input umoD pattern. The combination of IPTG and arabinose presence

216 resulted in a similar pattern, with narrower inner rings giving way to wider outer rings, but with

217 smaller colonies at 24 hours and asymmetric ring boundaries compared to those formed with

218 arabinose alone.

220 To characterize the cheW and $u m o D$ combination patterns in more detail, a dataset of plate 221 images at IPTG concentrations of $0,2.5$, and $5 \mathrm{mM}$ combined with arabinose at $0 \%, 0.1 \%$, and $2220.2 \%$ was created. The average percent of the plate covered by the colony at each condition 223 decreased with increasing IPTG and increased with the addition of arabinose (Fig. 4d). However, 224 increase of arabinose from $0.1 \%$ to $0.2 \%$ had little effect on the colony area except at $2.5 \mathrm{mM}$ 225 IPTG (Fig. S9). Similarly, average radial CV as a measure of colony asymmetry increased with 226 the induced expression of cheW, but decreased with the addition of arabinose inducing umoD 227 expression (Fig. 4e, S9).

As done previously for the single-input strains, a set of standard measurements was then taken

230 on each image in the dataset, and a 9-class multinomial regression model was fit on the output

231 (Fig. S10). The model performed poorly, predicting almost all images as $0 \%$ arabinose, and the 232 maximum AUC achieved was only 0.72. This result suggested that the two-input strain's patterns, 233 involving interdependent swarm genes, were too complex for the previous regression-based 234 decoding method. However, the ease of distinguishing the patterns by human eye suggested that 
235 the application of deep learning methods for image classification could prove useful for decoding 236 the patterns. In particular, deep convolutional neural networks (CNNs) have clear applicability and 237 have not yet been used to characterize macroscale bacterial colony patterns. CNNs can learn to 238 extract salient features from bacterial images and classify patterns to predict the image class ${ }^{41}$.

We fine-tuned models including ResNet and the Google Inception V3 networks to classify images in the dataset into one of the nine classes (details in Methods). The models were pre-trained on

242 ImageNet data, a common strategy in deep learning (Fig. S11) ${ }^{42}$. Here, the fine-tuned Google 243 InceptionV3 model was able to successfully classify the majority of our images (Fig. 4f). An ROC 244 curve was calculated (see Methods) and the AUC was 0.96, a noticeable improvement from the 245 multinomial regression model. Such models can also be characterized by "top-3" accuracy, i.e., 246 when used to predict the three most likely classes of an image, whether one of the three is the correct class; the fine-tuned model achieved a top-3 accuracy of 0.98 . We observed that intermediate concentrations of IPTG and arabinose reduced the model's accuracy due to some bimodality in pattern formation (Fig. 4f). Visualizing the pixel attributions of the model indicated the inoculum and inner rings had a large impact on the predictions, suggesting that these areas of the pattern were most affected by the induced expression of the different swarm genes (Fig. decoding may be possible after just a few hours of growth, rather than needing to wait 24 hours until the full plate is covered. Overall, these results suggest that our system can be used to encode and decode multiple inputs, and that the use of deep networks along with transfer learning will enable decoding of complex pattern feature changes.

\section{Multi-condition pattern segmentation and information decoding with deep learning}

We next sought to determine whether an engineered strain could record changes in the environment taking place during pattern formation and how these changes could be decoded from the endpoint pattern, similar to the analysis of rings in a tree ${ }^{23}$. We used the flgM strain, which we had observed to form two strikingly different patterns in the presence of $10 \mathrm{mM}$ IPTG in the incubator vs on the benchtop: a swarming-inhibited, ruffled, dense pattern in the incubator at $37^{\circ} \mathrm{C}$, and a wide-ring, symmetric, less dense pattern on the benchtop at $\sim 25^{\circ} \mathrm{C}$ (Fig. S11d). After inoculation, plates were first placed in one condition; after some time, plates were switched to a second condition, and certain plates were switched a third time before the endpoint scans were captured (Fig. 4h). Plates were scanned before each switch, creating a dataset of 21 images.

268 Representative pattern images are shown in Fig. 4i. This shift in environmental conditions 
resulted in the formation of rings alternating between indistinct, radially symmetric, wider rings and dense, asymmetrical, narrow rings, visible as bands on the polar-transformed images (Fig. 4j). In general, denser regions corresponded to incubator growth, while fainter regions with wider rings corresponded to benchtop growth.

To decode these alternating ring patterns, we manually annotated the dataset, creating ground

275 truth masks of the boundaries marking the shift in the pattern corresponding to a shift in the 276 environment. We then trained a U-Net model, a type of network frequently used for segmentation 277 problems, pretrained on ImageNet to predict these boundaries given an input pattern image 278 (details in Methods). Our model achieved above 95\% training and validation accuracy and above $27990 \%$ recall within the first 25 epochs of training, showing that it could learn the features within the dataset (Fig. S11e). Application of the trained model to previously unseen images resulted in specific prediction of boundaries matching the ground truth, and noticeably did not simply highlight all ring boundaries (Fig. 4j). In future, these predicted boundaries could be used to back-calculate the time at which a given perturbation was experienced, by generating prior control measurements of the time of formation of rings at different conditions. Taken together, these results demonstrated that our approach could be used to decode information about changing environment from the engineered strains' patterns.

\section{Discussion}

We have developed a proof-of-concept approach to engineering spatial patterns in $P$. mirabilis for information encoding and decoding. To date, bottom-up efforts to control spatiotemporal behaviors in microbial synthetic biology have required complex genetic circuits, used $E$. coli strains with liquid media, or required externally pre-patterned cues ${ }^{43-45}$. While there have been recent advances in encoding information in DNA and fluorescent bacterial colonies, there has not yet been an attempt to apply macroscopic pattern engineering for encoding information ${ }^{17,21,46}$. The approach described here takes advantage of the natural pattern formation capabilities of $P$. mirabilis on solid agar coupled with synthetic biological engineering approaches to modulate durable swarm patterns. We constructed genetic circuit variants with swarming-related genes and developed automated approaches for decoding information by quantifying aspects beyond typical colony radius measurements, such as colony asymmetry, swarming speed, frequency spectrum, and inoculum border distinctness. We then expanded to a dual-input system to sense two inducers, and trained a deep learning classifier to decode its patterns; while some works have begun to apply deep learning for segmentation of macroscale colonies, and in several cases for 
microscopic cell segmentation or classification of smaller colonies, our work represents a new application for classification, that of complex macroscale colony patterns ${ }^{47-51}$. At the same time, the macroscale patterns had many attributes distinguishable by eye, which could enhance the applicability of this system.

Since external conditions do affect pattern formation, a practical consideration for use of this system is to reliably produce robust patterns in differing laboratory or field conditions. We envision the use of this platform with side-by-side controls not exposed to the environment or input of interest, such that relative differences in pattern changes could be recorded. Additionally, future versions of the current system could include construction of knockout strains as well as chromosomal integration of promoter systems, which may allow for tighter control over the final pattern. In particular, for the dual-input strain, at the intermediate condition (2.5 mM IPTG and $0.1 \%$ arabinose), two distinct groups of patterns emerged, one in which colonies were small and dense, and one in which colonies swarmed almost to the edge of the plate. This stochasticity could possibly be reduced in future with further engineering, a different combination of genes or a different range of concentrations of inputs, which in turn can allow the decoding models to achieve higher accuracy. Enhanced imaging approaches such as incorporating a pigment into to the swarm medium or using pigment-producing strains may also improve accuracy. Further development of algorithms for image processing will benefit from the training and application of deep learning models for segmentation of colony and ring boundaries, such as the pipeline we have recently developed ${ }^{48,52}$. Additionally, the application of increasingly sophisticated computational approaches for modeling and machine learning-based classification will allow for the use of more complex spatiotemporal patterns ${ }^{48}$. Such models can be incorporated into easyto-run computer or mobile applications, and optimized for use with cell phone camera-images, allowing on-the-go analysis with inexpensive technologies. While we aimed to standardize our data acquisition method so that lighting, image size, and other factors would be constant throughout the datasets, these aspects can be intentionally varied to capture a more diverse dataset, which could help in developing models for application in a broader range of settings. several directions. The approach could be used to explore other inputs such as light, radiation, or gaseous molecules, or to develop a longer running recorder for changes in temperature or air quality. Other swarming species with natural swarming properties could be manipulated such as Pseudomonas aeruginosa, Paenibacillus vortex, or Bacillus subtilis ${ }^{2,3,53}$. Controlling swarming 
337 behaviors by engineering bacteria can enable multiple applications, ranging from bacteria drug 338 delivery to living material assembly. The approaches developed here can in turn shed light on $P$. 339 mirabilis growth dynamics and virulence, and be applied to understanding the coordinated and 340 emergent behaviors of microbes. 


\section{Methods}

Bacterial strains and growth conditions. Proteus mirabilis (ATCC 7002) was kindly provided by Dr. Philip Rather. Escherichia coli Mach1 for cloning was purchased from Fisher. P. mirabilis and E. coli were cultured in Luria-Bertani (LB) media (Sigma-Aldrich) supplemented with $50 \mu \mathrm{g}$ $\mathrm{ml}^{-1}$ kanamycin, respectively. P. mirabilis was grown on either $3 \%$ or $1.5 \%$ agar to suppress or allow for swarming, except for time-lapse assays as indicated.

Competent cell preparation. P. mirabilis (PM7002) cells and E. coli (Mach1) were made electrocompetent as follows. A fresh 2-mL overnight culture was subcultured 1:100 in $50 \mathrm{~mL}$ LB media, then grown at $30^{\circ} \mathrm{C}$ with shaking until logarithmic growth phase was reached, indicated when the optical density at $600 \mathrm{~nm}\left(\mathrm{OD}_{600}\right)$ was $0.4-0.6$. Growth was stopped by incubation of the culture on ice for 15 minutes. Cells were then pelleted by centrifuging for 10 minutes at $4{ }^{\circ} \mathrm{C}$ and $3000 \mathrm{rpm}$. After decanting, the pellet was washed three times in either $50 \mathrm{~mL}$ ice-cold filtersterilized $10 \%$ glycerol $(P$. mirabilis) or $50 \mathrm{~mL}$ ice-cold filter-sterilized water $(E$. coli), then resuspended in $220 \mu \mathrm{L} 10 \%$ glycerol. $50 \mu \mathrm{L}$ aliquots were stored in $-80^{\circ} \mathrm{C}$.

Strain construction. The previously constructed pZE24 (pLacGFP pConstLaclQ) plasmid, containing the ColE1 origin of replication and a kanamycin resistance cassette, was used as the backbone for the inducible swarming plasmids. Plasmids and chromosomal P. mirabilis DNA were prepared using standard procedures (Quiagen). Swarming gene sequences were obtained from GenBank (JOVJ00000000.1) and Gibson primers were designed (Eton) to amplify the genes from the chromosomal DNA via PCR (Phusion) ${ }^{54}$. A set of swarming plasmids were constructed using Gibson Assembly and standard restriction digest and ligation cloning to replace the gfp gene with the appropriate swarming gene. For plasmids which additionally contained $p B A D$-araC, the operon was obtained from the pBADmCherry-pConstAra plasmid (ATCC54630). After cloning plasmids into Mach1 E. coli, clones were verified via colony PCR (Phusion) and sequencing (Eton). Clones were then grown at $37^{\circ} \mathrm{C}$ with shaking overnight before being stored in $50 \%$ glycerol at $-80^{\circ} \mathrm{C}$. All plasmids and strains are listed in Tables $\mathbf{S 1}$ and $\mathbf{S 2}$; plasmid maps are shown in Fig. S12.

P. mirabilis transformation. Plasmid DNA was introduced into $P$. mirabilis competent cells as

373 follows. $50 \mu \mathrm{L}$ aliquots of competent cells were thawed on ice for 10 minutes. DNA was added to 374 the cells (200-400 ng DNA in a volume of 1-5 $\mu \mathrm{L}$ per aliquot). The mixture was then incubated on 
375 ice for one hour. Cells were electroporated in prechilled electroporation $0.1 \mathrm{~cm}$ electrode gap cuvettes using a Bio-Rad MicroPulser set to E1 setting $(1.8 \mathrm{kV})$ for bacterial electroporation. Cells were recovered by adding $1 \mathrm{~mL}$ prewarmed SOC media and incubated with shaking at $37^{\circ} \mathrm{C}$ for 3 hours. The cells were pelleted by centrifugation for 10 minutes at $4^{\circ} \mathrm{C}$ and $3000 \mathrm{rpm}$, and 700 $\mu \mathrm{l}$ of the supernatant was decanted before resuspension in the remaining $300 \mu \mathrm{l}$. The cells were then plated on pre-warmed 3\% LB agar plates with antibiotics as necessary and incubated at $37^{\circ} \mathrm{C}$ for 22-24 hours. Single colonies were inoculated and fresh overnight cultures were stored in $50 \%$ glycerol at $-80^{\circ} \mathrm{C}$.

Bacterial growth and swarm assay. Overnight liquid bacterial cultures were prepared by inoculating LB media with cells from the $-80^{\circ} \mathrm{C}$ glycerol stocks and supplementing with $50 \mu \mathrm{ml}^{-}$

${ }^{1}$ kanamycin as appropriate. Cultures were incubated at $37^{\circ} \mathrm{C}$ with shaking for $12-16$ hours. The $\mathrm{OD}_{600}$ of each culture was measured and normalized to 1.0 by dilution with LB media. Swarm assays were optimized from a protocol adapted from literature. A study to develop standard conditions is shown in Fig. S1. Precise maintenance of the selected conditions was necessary to achieve comparable results ${ }^{55}$. 1.5\% agar (or, where indicated, $1.3 \%$ agar) was autoclaved, then cooled to $50-55^{\circ} \mathrm{C}$ with stirring. $5 \mathrm{\mu g} \mathrm{ml}^{-1}$ kanamycin, IPTG and/or arabinose were then added as necessary. $15 \mathrm{~mL}$ agar was poured in each 100x15 mm Petri dish and left to solidify partially uncovered under an open flame for exactly 30 minutes. $2 \mu \mathrm{L}$ of the previously diluted liquid culture was inoculated on the center of each Petri dish and dried for 15 minutes partially uncovered under open flame. The plates were incubated at $37^{\circ} \mathrm{C}$ for 24 hours, then individually imaged using a scanner (Epson Perfection V800 Photo Scanner) set to 48-bit Color and $400 \mathrm{dpi}$, with the lid off and colony side facing up. The scanner was kept on the benchtop and room lighting was similar during all experiments; other settings of the scanner were also kept constant between experiments. Incubator humidity typically varied between $50-80 \%$ during the course of experiments.

Time-lapses. For time-lapses on the benchtop (room temperature), up to six plates with $20 \mathrm{~mL}$ $4031.3 \%$ LB agar were inoculated and placed on the flatbed scanner using the previously described 404 settings, and kept upside down to prevent condensation and with lids on to prevent contamination. 405 A custom AppleScript was written to scan plates every 10 minutes for a pre-set length of time 406 (typically $48-72$ hours). Typical benchtop conditions were $25^{\circ} \mathrm{C}$ and $40-50 \%$ humidity. 
Computational methods. Measurements of colony features were taken using MATLAB (Mathworks) image and signal processing functions. Images were preprocessed by conversion to grayscale; the plate rim was removed using the imfindcircles (based on a Hough transform) and regionprops functions, then the image was thresholded to find the colony's center inoculum,

412 typically easily identified by its dark boundary. Upon finding the center point, the colony was 413 unrolled or "flattened" using a Cartesian to polar transformation and the scattered interpolant 414 function, and resized to $1000 \times 1000$ pixels for ease of scaling analysis for the full dataset. The 415 colony rim was also masked out (set to white). Radial profiles could then be easily generated by 416 averaging the pixel intensity across each row of the image. The ring widths in Fig. 1 were 417 calculated by using 1-D Fourier/inverse Fourier transformation on the radial profile of each image 418 of interest to filter out noise and by subsequent peak-finding. The ring widths of a single image's 419 radial profile were averaged to generate the individual measurements in Fig. 1 e.

The colors selected for plots of the different strains in figures 2-4 were derived from a previously developed "bright" color scheme ${ }^{56}$. Where described, local CV was calculated by moving a sliding window region of width 10 pixels across each row and calculating the $\mathrm{CV}$ within it, then taking the average of these calculated CVs over the whole image. Mean CV was calculated by obtaining the $\mathrm{CV}$ across each row, then averaging over all the rows. The inoculum edge intensity was measured for a given image as follows: the image was smoothed using the movmean function with averaging applied in 25-pixel windows horizontally. For each individual column of the smoothed image, the minimum value between the $15^{\text {th }}$ and $60^{\text {th }}$ rows (ie, in the region of the inoculum border) was subtracted from the maximum value in that region. The average over all the columns was then taken (calculation schematics in Fig. S5a).

For certain measurements, a mask of the colony region was desired. A custom algorithm was developed using image processing functions in MATLAB. Briefly, a set of filters were applied to

434 reduce local noise such as dust and scratches, then adaptive histogram equalization was applied 435 to increase contrast. The entropyfilt function in MATLAB was applied and the output was 436 thresholded, then the difference between this output and the original image was taken in order to 437 sharpen the edges in the colony. The image was binarized and a series of morphological 438 operations including dilation, opening, and hole-filling were applied to obtain a mask of the colony. 439 The largest region was retained and all smaller regions were discarded. 
441 In order to analyze the time-lapses, a method to track a growing swarm colony was sought; such

442 methods have been of recent interest ${ }^{48,57}$. P. mirabilis presents a unique challenge in this area;

443 during its swarm phase, only a thin, almost transparent film of bacteria moves outwards, almost

444 indistinguishable from local variations in agar intensity. Thus, the swarm front is difficult to detect

445 with conventional thresholding-based or edge-detection algorithms which have been

446 implemented previously for analysis of other species ${ }^{58-60}$. The colony region isolation algorithm

447 described above also did not work on these images. The movement outwards on the plate (or

448 vertically down on the flattened images) over time is difficult and noisy to capture. Towards an

449 algorithm for tracking the swarm edge, each time-lapse image was first flattened as described

450 above. Each image was subtracted from the preceding image using the imabsdiff function. The

451 difference images were then averaged across columns, creating a radially averaged trajectory. In

452 brief, the findpeaks function was used on each timepoint's trajectory, using a custom algorithm

453 and manual parameter refinement to determine the location in which to seek the peak, and taking

454 advantage of the constraint that the colony edge would not move backwards over time. The user

455 could choose (1) the minimum possible prominence of the peaks and (2) the range to the right of

456 each previous peak in which the algorithm would seek the next timepoint's peak, and then the

457 algorithm would iterate over the whole time-lapse. The process would be repeated until the user

458 was satisfied with the visual overlay of identified peaks on the time-lapse heatmap. The obtained

459 colony front trajectory was then labeled using a custom algorithm involving the moving_polyfit

460 function, bwareaopen and bwlabel, from which the locations of the lag phase, swarm phases, and

461 consolidation phases were obtained ${ }^{61}$. In Fig. 3f, the cheW measurements were calculated by

462 discarding the first and last consolidation phases and measuring the length of only the middle

463 consolidation phases.

Statistical tests were calculated and data was plotted either in MATLAB or in Python. Latex tables were generated using Overleaf. Multinomial regression models were fit to the measurements using the mnrfit function in MATLAB, returning the coefficients and p-values in Fig. S5b. For the

468 single input strain data in Fig. 2h, each flattened image was divided into four sectors (each 250 469 pixels wide) and measurements were taken on each sector to increase the number of 470 measurements available, so that the model fitting could converge. The models were evaluated 471 using the multiClassAUC function, which implements the Hand and Till function for area under 472 the curve for multi-class problems ${ }^{62}$. Machine learning models were implemented in Tensorflow 473 and Pytorch, with manual annotation of the flgM ground truth segmentation done using the 474 LabelMe program ${ }^{63}$. Attributions in Fig. $\mathbf{4 g}$ were calculated following the Integrated Gradients 
475 method of Sundarajan et $\mathrm{al}^{64}$. Fine tuning of the pre-trained models for classification of the dual-

476 input strain was done with on-the-fly augmentation of the dataset, using random rotations, 477 translations, and horizontal flips ${ }^{65-67}$. For the U-Net segmentation work, a VGG-11 Encoder pre478 trained on ImageNet was used ${ }^{68-70}$. Predicted masks from the U-Net model were postprocessed 479 using standard methods. In brief, the predicted masks were dilated to ensure a given boundary 480 was fully connected, then opened to remove any small instances of detected noise. The cleaned 481 masks were then skeletonized to obtain single-pixel thick boundaries for evaluation of metrics 482 such as accuracy. Finally, a flat line-shaped structuring element was applied to dilate near the left 483 and right edges to re-connect the boundaries with these edges. For the visualization in Fig. 4i, 484 masks were dilated with a disk element for better visibility.

Acknowledgments. We thank Professor Martina Pavlicova for helpful discussion of the statistics methods used. We thank the members of the Danino lab for review of the manuscript. We thank R. Minyety for assisting in time-lapse experiments. Funding: This work was supported by an NSF CAREER Award (1847356) and NSF Graduate Research Fellowship (A.D.). Author contributions: A.D. and T.D. conceived and designed the study. A.D., M.S., R.T., and S.M. performed experiments and constructed the dataset. A.D. and M.S. performed the computational analysis. A.D., M.S., and A.D. (Berkeley) carried out the deep-learning work with input from J.G. and A.L. A.D. and T.D. wrote the original manuscript draft and A.D., M.S., and T.D. edited the manuscript with input from all authors. Competing interests: A.D., M.S., J. G., A. L., and T.D. have filed a provisional patent application with the US Patent and Trademark Office related to this work. Data and materials availability: All data is available upon reasonable request. Correspondence and request for materials should be addressed to T.D. Code Availability: All code is proprietary and managed by the Columbia Technology Ventures Office of Intellectual Property. They are available from the corresponding author T.D. upon reasonable request, after permission from the Columbia Technology Ventures Office of Intellectual Property. 
a
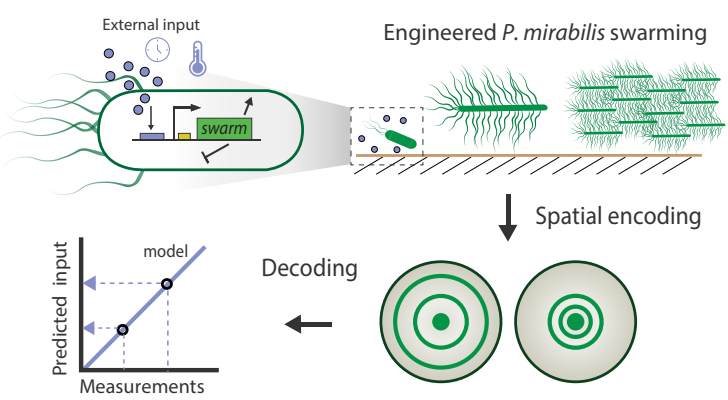

Decoding
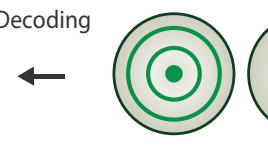

C Trajectory at $0.5 \mathrm{mMIPTG}$
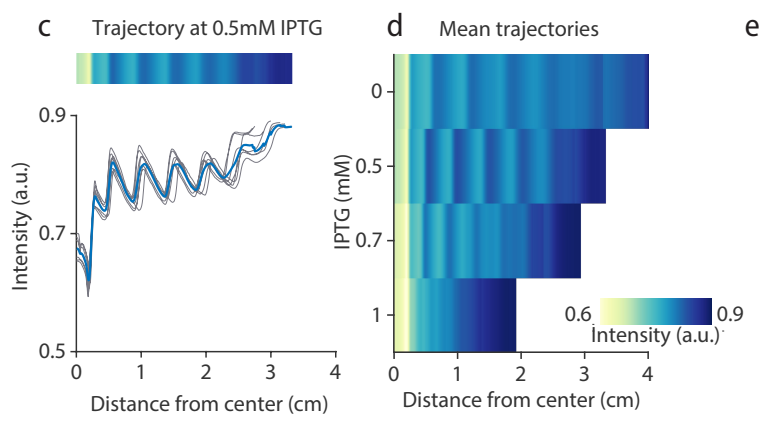
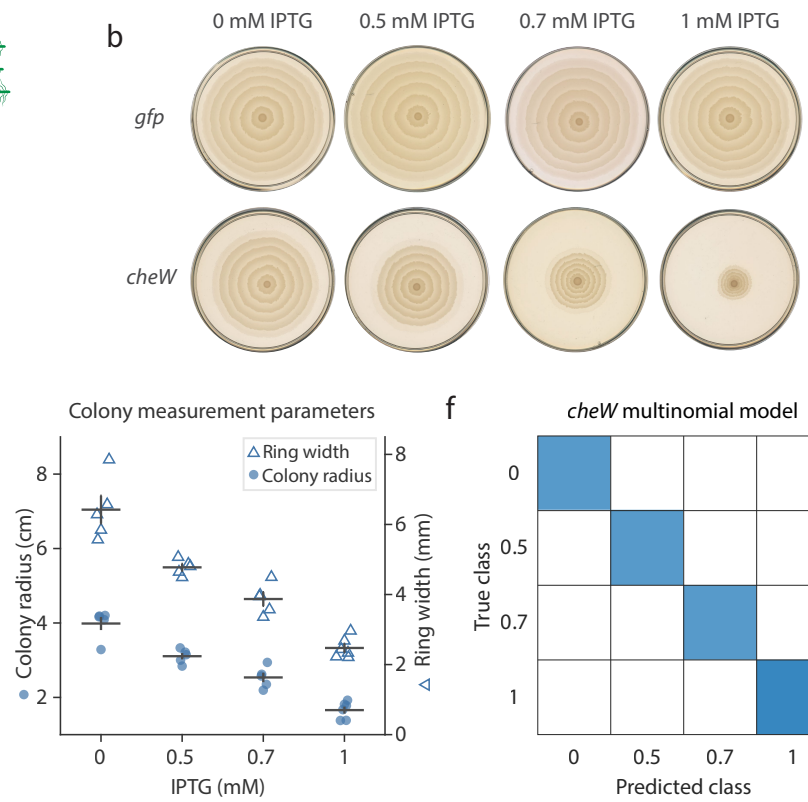

506

507

508

Figure 1: Engineered $\boldsymbol{P}$. mirabilis swarm patterns as spatiotemporal records. a. Wild type $P$. mirabilis cells undergo oscillatory swarming on solid agar to grow into a characteristic bullseye colony via elongation, hyperflagellation, and raft formation. $P$. mirabilis is engineered with an externally inducible genetic circuit driving swarming-related genes to modify the macroscale pattern output, which can then be decoded using quantitative methods to predict the input conditions. b. Representative images of colony patterns formed by a strain containing a control circuit with green fluorescent protein ( $g f p$ ) (top) compared to a circuit with the chemotaxis gene cheW (bottom), grown for 24 hours on agar supplemented with various IPTG concentrations. c. The cheW colony pattern is distilled into radially averaged pixel intensity profiles, with distinct peaks matching low-density ring boundaries when plotted as a heatmap or line plot. The blue line denotes the mean profile of the individual plates (each gray line represents one plate). d. Heatmaps of average cheW profiles at varying IPTG concentration ( $n=5$ plates at each condition except $1 \mathrm{mM}$ IPTG $(n=6)$ ). Colormap is on same scale for $(\mathbf{c})$ and $(\mathbf{d})$. e. Radii of the colonies plotted by IPTG concentration after 24 hours (filled circles) and calculated ring width (empty triangles), derived from Fourier analysis of the radially averaged profiles of individual images. The mean and standard error of the mean (SEM) are shown in black. f. A multinomial model was fit to the measurements in (e), with predicted IPTG concentration as the output variable. The model's predictions for each plate shown in (e) are shown as a confusion matrix. Color reflects $\mathrm{n}$ per square (same as listed in (d); white squares represent 0 ). 


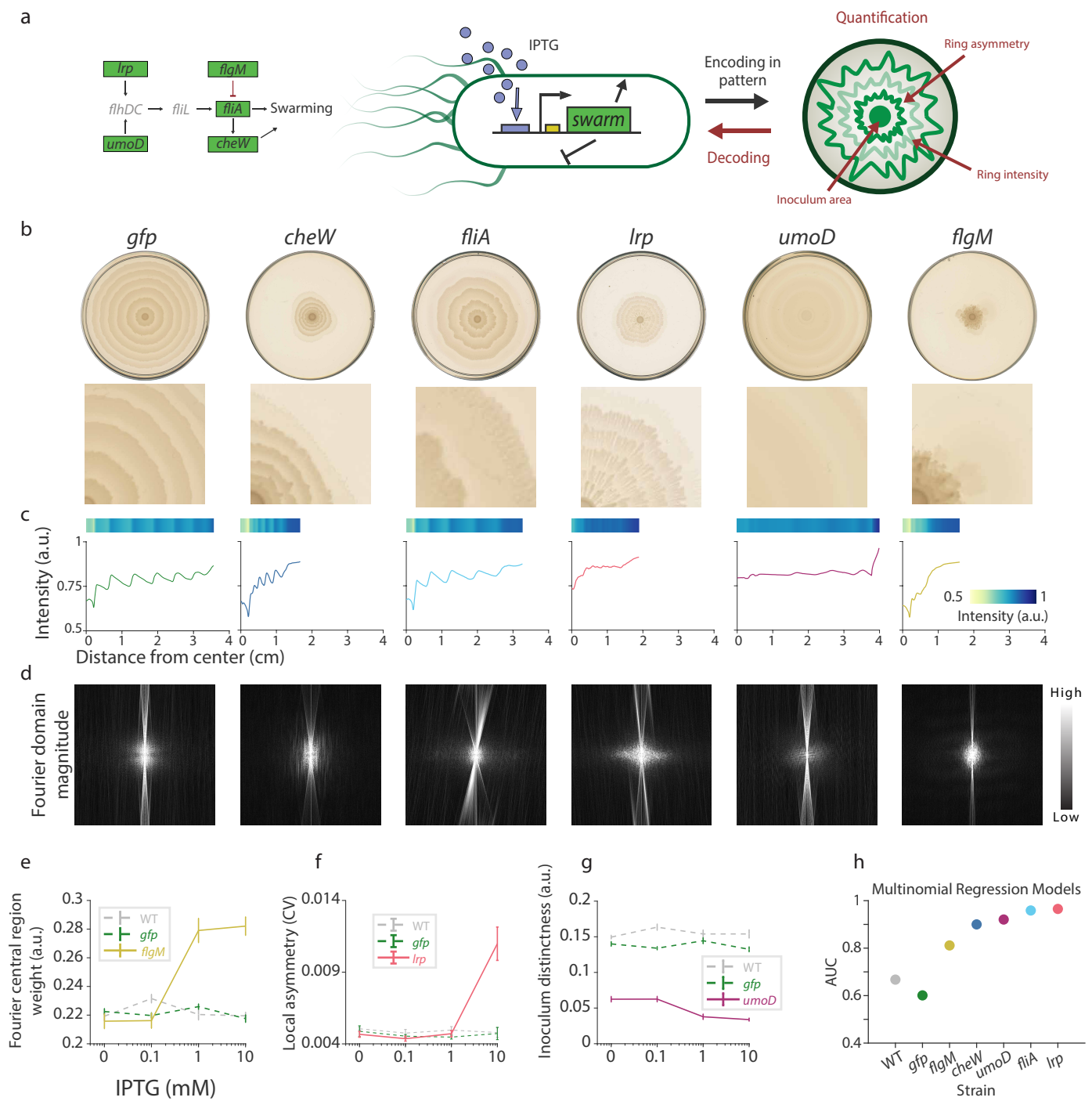

Figure 2: Modulation of swarm genes in engineered $\boldsymbol{P}$. mirabilis results in quantifiable changes to distinct spatial pattern features. a. Candidate genes involved in $P$. mirabilis swarming pathway were chosen for construction of inducible strains. The patterns in the presence of inducer were characterized by growth on IPTG-supplemented solid agar, then by specific feature measurements used to recover the inducer concentration. b. Characteristic patterns of engineered strains in the presence of IPTG and closeups of pattern features. c. For each induced strain, heatmaps and plots of radially averaged intensity profiles across the colony for the representative images in (b). d. Fourier transforms of the polar images visualize the magnitudes of the intensity frequencies of each induced strain. e-g. Quantification of aspects of colony patterns of engineered strains at increasing IPTG concentrations. All strains had at least $n=3$ plates measured at each IPTG concentration. Error bars represent standard error of the mean (SEM). Details can be found in Methods. e. Intensity of central region compared to total intensity of the Fourier transform of the polar image. $\mathbf{f}$. Local radial coefficient of variation (CV), which increases with colony asymmetry. g. Change in intensity from the densest edge of the inoculum (innermost circular region of colony) to the low-density region immediately surrounding it, i.e., distinctness of the inoculum edge, where low values correspond to less distinct edges. $\mathbf{h}$. Area under the curve (AUC) of multinomial regression models for predicting IPTG concentration, fit with specific pattern measurements for each strain. 


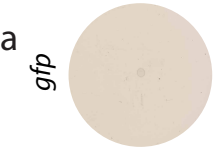

$5 \mathrm{~h}$

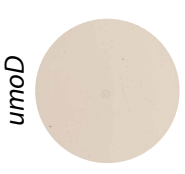

$9 \mathrm{~h}$

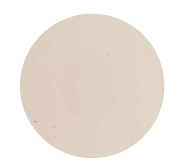

C

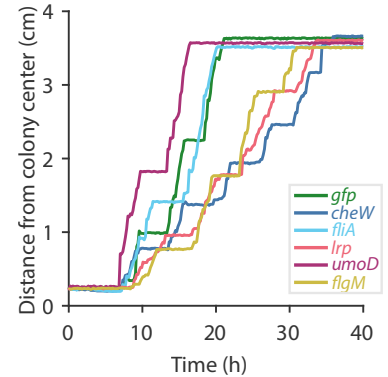

f

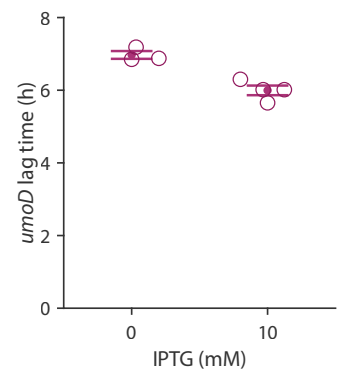

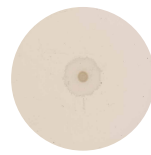

$13 \mathrm{~h}$

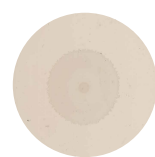

d
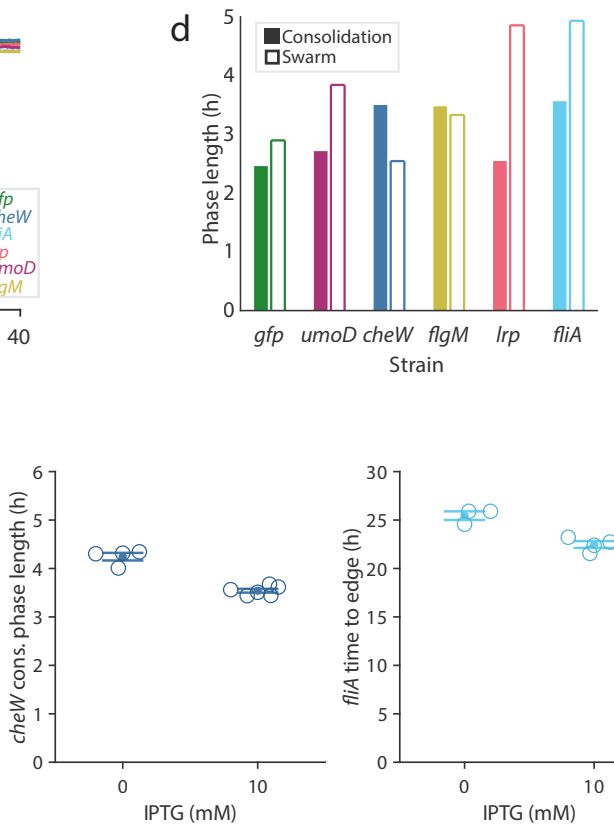

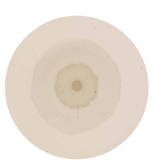

$18 \mathrm{~h}$
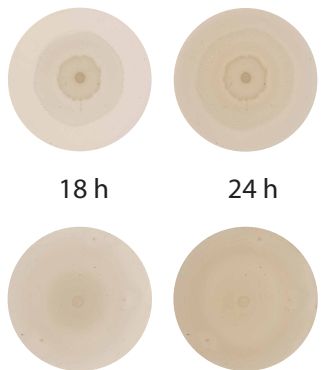

$24 \mathrm{~h}$

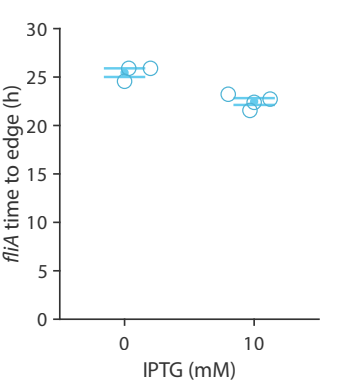

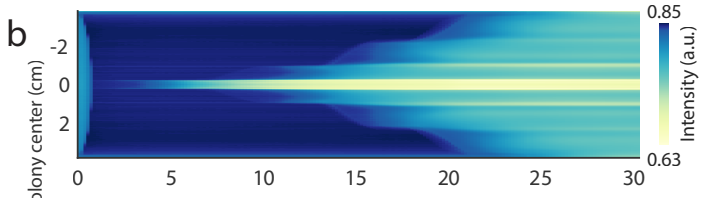
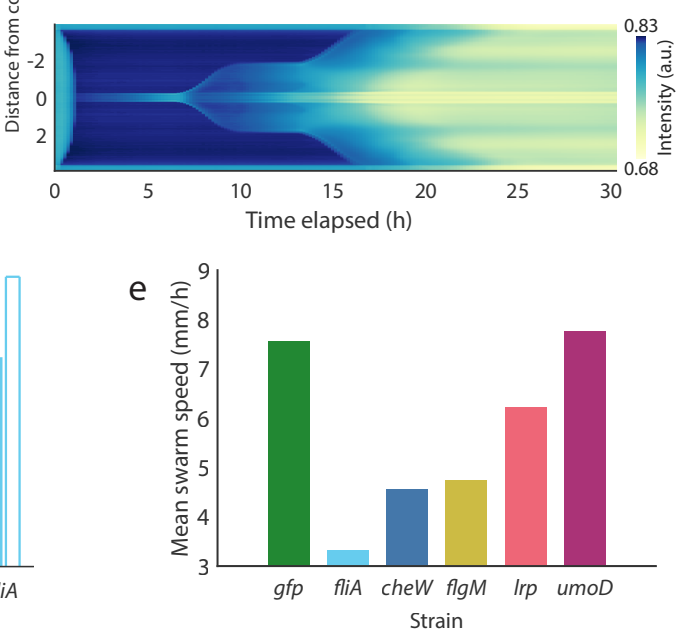

9

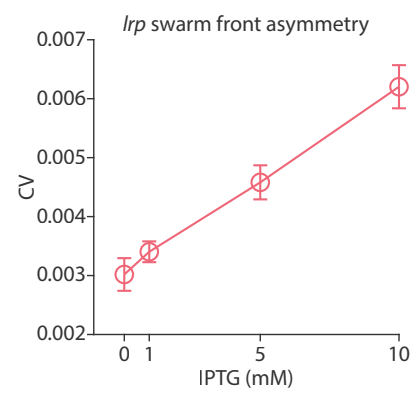

Figure 3: Dynamics of engineered $P$. mirabilis pattern formation. a. Time-lapse of $P$. mirabilis with inducible $u m o D$ expression or inducible gfp expression (control). Plates contained $20 \mathrm{~mL}$ $1.3 \%$ agar with $10 \mathrm{mM}$ IPTG. b. Heatmap visualizations of swarming pattern development from center of plates $(0 \mathrm{~cm}$ on left axis) to edge (top and bottom edges) for each image in the timelapses in (a). Radially averaged pixel intensity, a proxy for local colony density, at each location on plate is represented by heatmap color, with blue indicating least dense and yellow indicating most dense regions. Active regions and time periods of colony expansion via swarming are visible as faint blue diagonal edges. Consolidation phases appear as horizontal edges corresponding with increasing density (lighter colors) within the colony. c. Colony front distance from center plotted as a function of time for a single time-lapse of six plates. All plates contained $10 \mathrm{mM} \mathrm{IPTG.}$ d. Mean consolidation (filled bars, left) and swarm (outlined bars, right) phase lengths calculated from the trajectories in (d). e. Mean of the swarm speeds for each strain in the same time-lapse. f. Measurements of dynamic features at 0 vs $10 \mathrm{mM} \mathrm{IPTG}$ for the indicated strains. Each condition and strain was tested on at least $n=3$ separate plates. All plots represent a significant difference between induced and uninduced conditions ( $p$-values from a 2-sample t-test were $0.003,2 e-5$, 0.003 for the plots of $u m o D$, cheW, and fliA respectively.) g. The local CV of the swarm front for phases at 5 and $10 \mathrm{mM} \mathrm{IPTG)}$ at each given IPTG. Error bars in (f) and (g) represent SEM. 

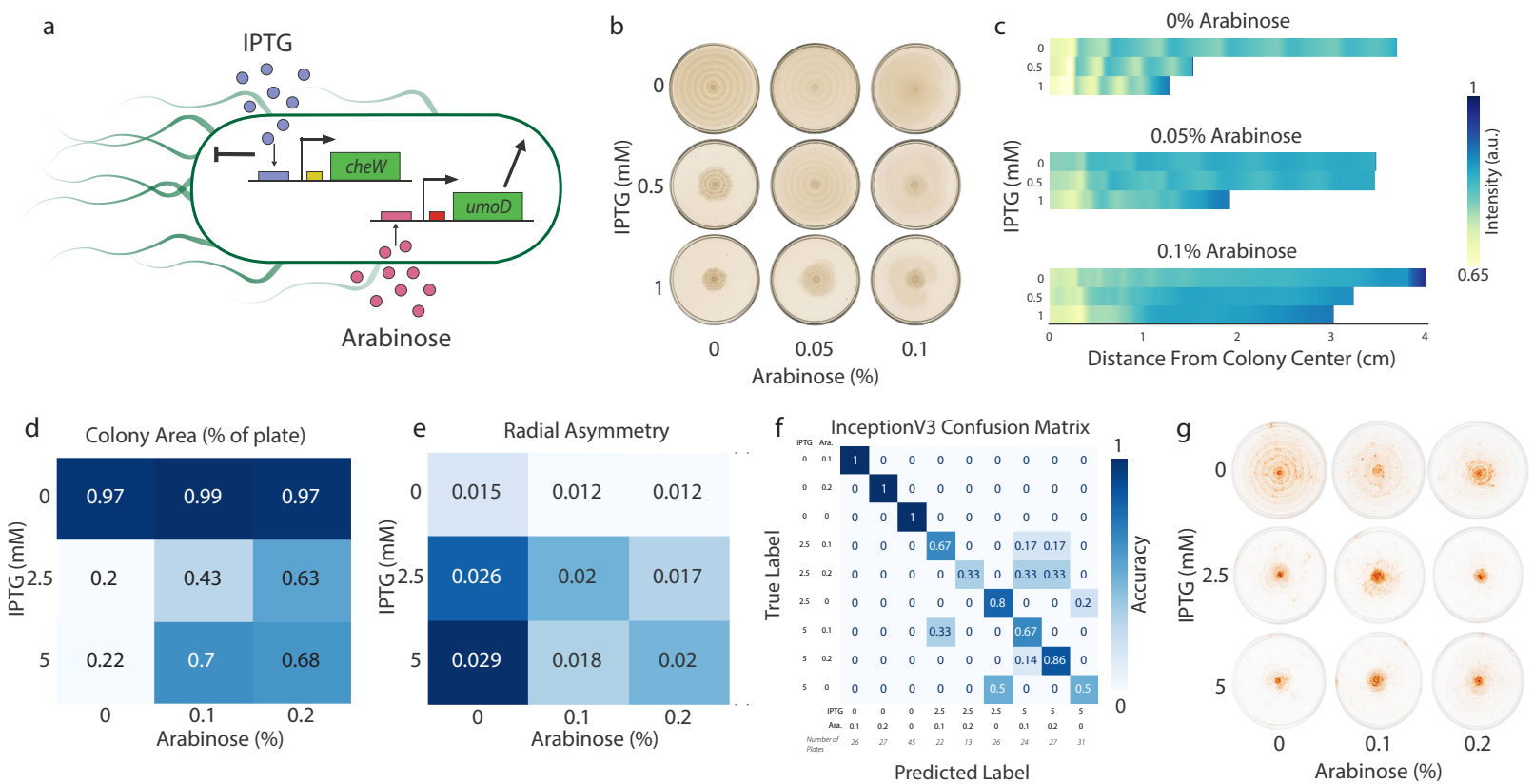

h

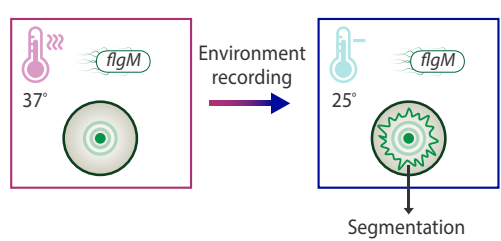

i

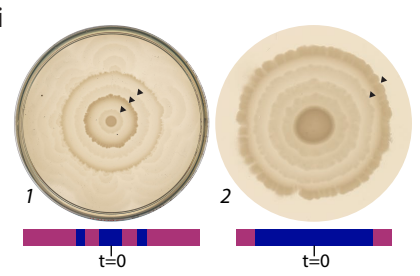

j

Segmentation of colony boundary Ground Truth

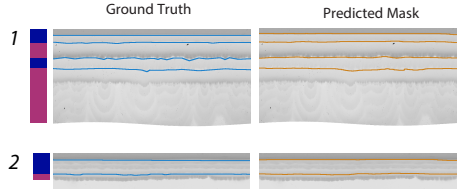

Figure 4: Multi-condition pattern encoding and deep-learning models for decoding. a. Dual input swarming strain with IPTG-inducible expression of cheW and arabinose-inducible expression of umoD. b. Representative images of colony patterns produced by the dual cheW/umoD strain in response to combinations of IPTG and arabinose. c. Heatmaps of radially averaged profiles of the patterns in (b) are shown. d-e. Mean colony area (calculated as percent of agar area in flattened image) and coefficient of variation for all plates ( $n>=14)$ at each combination of IPTG and arabinose. f. Confusion matrix for the InceptionV3 model's accuracy of predicting combinations of IPTG and arabinose concentrations from endpoint patterns unseen during training. Total available images per class shown below matrix; an 80/20 train/test split was used. Numbers on matrix represent fraction of test images per true class. g. Visualization of the pixel attributions from the InceptionV3 model for representative, correctly-predicted images of each class. Darker orange represents higher weight of that pixel on the final prediction. $\mathbf{h}$. Schematic of encoding of environmental changes within developing flgM pattern. i. Example patterns of the flgM strain grown with $10 \mathrm{mM}$ IPTG and moved between the benchtop and incubator. Arrows mark boundaries between regions of the pattern formed in different conditions, i.e., the location of the colony edge at the time of a switch in conditions. $\mathbf{j}$. Examples of the predicted boundary masks generated by the trained U-Net compared with ground truth annotations for the pattern images shown in (i), which were unseen during training. 
588

589

590

591

592

593

594

595

596

597

598

599

600

601

602

603

604

605

606

607

608

609

610

611

612

613

614

615

616

617

618

619

620

621

622

623

624

625

626

627

628

629

630

631

632

633

634

635

636

\section{References}

1. Kearns, D.B., A field guide to bacterial swarming motility. Nature Reviews Microbiology, 2010. 8(9): p. 634.

2. Ingham, C.J. and E. Ben Jacob, Swarming and complex pattern formation in Paenibacillus vortex studied by imaging and tracking cells. BMC microbiology, 2008. 8: p. 36-36.

3. Kearns, D.B. and R. Losick, Swarming motility in undomesticated Bacillus subtilis. Mol Microbiol, 2003. 49(3): p. 581-90.

4. Kohler, T., L.K. Curty, F. Barja, C. van Delden, and J.C. Pechere, Swarming of Pseudomonas aeruginosa is dependent on cell-to-cell signaling and requires flagella and pili. J Bacteriol, 2000. 182(21): p. 5990-6.

5. Rauprich, O., M. Matsushita, C.J. Weijer, F. Siegert, S.E. Esipov, and J.A. Shapiro, Periodic phenomena in Proteus mirabilis swarm colony development. Journal of Bacteriology, 1996. 178(22): p. 6525.

6. Schuerle, S., A.P. Soleimany, T. Yeh, G. Anand, M. Häberli, H. Fleming, N. Mirkhani, F. Qiu, S. Hauert, and X. Wang, Synthetic and living micropropellers for convectionenhanced nanoparticle transport. Science advances, 2019. 5(4): p. eaav4803.

7. Li, S., R. Batra, D. Brown, H.-D. Chang, N. Ranganathan, C. Hoberman, D. Rus, and H. Lipson, Particle robotics based on statistical mechanics of loosely coupled components. Nature, 2019. 567(7748): p. 361-365.

8. Rubenstein, M., A. Cornejo, and R. Nagpal, Programmable self-assembly in a thousandrobot swarm. Science, 2014. 345(6198): p. 795-799.

9. Kearns, D.B., A field guide to bacterial swarming motility. Nature reviews. Microbiology, 2010. 8(9): p. 634-644.

10. Fujikawa, H. and M. Matsushita, Fractal Growth of Bacillus subtilis on Agar Plates. Journal of the Physical Society of Japan, 1989. 58(11): p. 3875-3878.

11. Prindle, A., P. Samayoa, I. Razinkov, T. Danino, L.S. Tsimring, and J. Hasty, A sensing array of radically coupled genetic 'biopixels'. Nature, 2012. 481(7379): p. 39-44.

12. Payne, S., B. Li, Y. Cao, D. Schaeffer, M.D. Ryser, and L. You, Temporal control of selforganized pattern formation without morphogen gradients in bacteria. Molecular systems biology, 2013. 9(1): p. 697.

13. Santos-Moreno, J. and Y. Schaerli, Using Synthetic Biology to Engineer Spatial Patterns. Advanced Biosystems, 2019. 3(4): p. 1800280.

14. Basu, S., Y. Gerchman, C.H. Collins, F.H. Arnold, and R. Weiss, A synthetic multicellular system for programmed pattern formation. Nature, 2005. 434(7037): p. 1130-1134.

15. Liu, C., X. Fu, L. Liu, X. Ren, C.K.L. Chau, S. Li, L. Xiang, H. Zeng, G. Chen, L.-H. Tang, P. Lenz, X. Cui, W. Huang, T. Hwa, and J.-D. Huang, Sequential Establishment of Stripe Patterns in an Expanding Cell Population. Science, 2011. 334(6053): p. 238-241.

16. Curatolo, A., N. Zhou, Y. Zhao, C. Liu, A. Daerr, J. Tailleur, and J. Huang, Cooperative pattern formation in multi-component bacterial systems through reciprocal motility regulation. Nature Physics, 2020. 16(11): p. 1152-1157.

17. Sheth, R.U., S.S. Yim, F.L. Wu, and H.H. Wang, Multiplex recording of cellular events over time on CRISPR biological tape. Science, 2017. 358(6369): p. 1457-1461.

18. Perli, S.D., C.H. Cui, and T.K. Lu, Continuous genetic recording with self-targeting CRISPR-Cas in human cells. Science, 2016. 353(6304).

19. Frieda, K.L., J.M. Linton, S. Hormoz, J. Choi, K.-H.K. Chow, Z.S. Singer, M.W. Budde, M.B. Elowitz, and L. Cai, Synthetic recording and in situ readout of lineage information in single cells. Nature, 2017. 541(7635): p. 107-111. 
637

638

639

640

641

642

643

644

645

646

647

648

649

650

651

652

653

654

655

656

657

658

659

660

661

662

663

664

665

666

667

668

669

670

671

672

673

674

675

676

677

678

679

680

681

682

683

684

685

686

687

20. Shipman, S.L., J. Nivala, J.D. Macklis, and G.M. Church, CRISPR-Cas encoding of a digital movie into the genomes of a population of living bacteria. Nature, 2017.

547(7663): p. 345-349.

21. Riglar, D.T., D.L. Richmond, L. Potvin-Trottier, A.A. Verdegaal, A.D. Naydich, S. Bakshi, E. Leoncini, L.G. Lyon, J. Paulsson, and P.A. Silver, Bacterial variability in the mammalian gut captured by a single-cell synthetic oscillator. Nature communications, 2019. 10(1): p. 1-12.

22. Schaffer, J.N. and M.M. Pearson, Proteus mirabilis and urinary tract infections, in Urinary Tract Infections: Molecular Pathogenesis and Clinical Management. 2017, ASM Press: Washington, DC. p. 383-433.

23. Cook, E.R. and N. Pederson, Uncertainty, emergence, and statistics in dendrochronology, in Dendroclimatology. 2011, Springer. p. 77-112.

24. Hauser, G., Uber Faulnisbakterien und deren Beziehung zur Septicamie. FGW Vogel, 1885.

25. Saak, C.C., K.A. Gibbs, and V.J. DiRita, The Self-Identity Protein IdsD Is Communicated between Cells in Swarming Proteus mirabilis Colonies. Journal of Bacteriology, 2016. 198(24): p. 3278-3286.

26. Fraser, G.M. and C. Hughes, Swarming motility. Curr Opin Microbiol, 1999. 2(6): p. 6305.

27. Clemmer, K.M. and P.N. Rather, Regulation of flhDC expression in Proteus mirabilis. Research in Microbiology, 2007. 158(3): p. 295-302.

28. Howery, K.E., E. Simsek, M. Kim, and P.N. Rather, Positive autoregulation of the flhDC operon in Proteus mirabilis. Res Microbiol, 2018. 169(4-5): p. 199-204.

29. Pearson, M.M., D.A. Rasko, S.N. Smith, and H.L. Mobley, Transcriptome of swarming Proteus mirabilis. Infect Immun, 2010. 78(6): p. 2834-45.

30. Şimşek, E., E. Dawson, P.N. Rather, and M. Kim, Spatial regulation of cell motility and its fitness effect in a surface-attached bacterial community. The ISME journal, 2021: $p$. $1-8$.

31. Armbruster, C.E., S.A. Hodges, and H.L. Mobley, Initiation of swarming motility by Proteus mirabilis occurs in response to specific cues present in urine and requires excess L-glutamine. Journal of bacteriology, 2013. 195(6): p. 1305-1319.

32. Little, K., J. Austerman, J. Zheng, and K.A. Gibbs, Cell shape and population migration are distinct steps of Proteus mirabilis swarming that are decoupled on high-percentage agar. Journal of bacteriology, 2019. 201(11): p. e00726-18.

33. Burall, L.S., J.M. Harro, X. Li, C.V. Lockatell, S.D. Himpsl, J.R. Hebel, D.E. Johnson, and H.L. Mobley, Proteus mirabilis genes that contribute to pathogenesis of urinary tract infection: identification of 25 signature-tagged mutants attenuated at least 100-fold. Infect Immun, 2004. 72(5): p. 2922-38.

34. Fraser, G.M., R.B. Furness, and C. Hughes, Swarming migration by Proteus and related bacteria. Prokaryotic Development, 1999: p. 379-401.

35. Huang, Z., X. Pan, N. Xu, and M. Guo, Bacterial chemotaxis coupling protein: Structure, function and diversity. Microbiological research, 2019. 219: p. 40-48.

36. Dufour, A., R.B. Furness, and C. Hughes, Novel genes that upregulate the Proteus mirabilis flhDC master operon controlling flagellar biogenesis and swarming. Molecular microbiology, 1998. 29(3): p. 741-751.

37. Hay, N.A., D.J. Tipper, D. Gygi, and C. Hughes, A nonswarming mutant of Proteus mirabilis lacks the Lrp global transcriptional regulator. Journal of Bacteriology, 1997. 179(15): p. 4741-4746.

38. Clemmer, K.M. and P.N. Rather, The Lon protease regulates swarming motility and virulence gene expression in Proteus mirabilis. Journal of Medical Microbiology, 2008. 57(8): p. 931-937. 
688

689

690

691

692

693

694

695

696

697

698

699

700

701

702

703

704

705

706

707

708

709

710

711

712

713

714

715

716

717

718

719

720

721

722

723

724

725

726

727

728

729

730

731

732

733

734

735

736

737

39. Gygi, D., G. Fraser, A. Dufour, and C. Hughes, A motile but non-swarming mutant of Proteus mirabilis lacks FlgN, a facilitator of flagella filament assembly. Mol Microbiol, 1997. 25(3): p. 597-604.

40. Morgenstein, R.M. and P.N. Rather, Role of the Umo proteins and the Rcs phosphorelay in the swarming motility of the wild type and an O-antigen (waaL) mutant of Proteus mirabilis. Journal of bacteriology, 2012. 194(3): p. 669-676.

41. LeCun, Y., B. Boser, J.S. Denker, D. Henderson, R.E. Howard, W. Hubbard, and L.D. Jackel, Backpropagation Applied to Handwritten Zip Code Recognition. Neural Computation, 1989. 1(4): p. 541-551.

42. Russakovsky, O., J. Deng, H. Su, J. Krause, S. Satheesh, S. Ma, Z. Huang, A. Karpathy, A. Khosla, and M. Bernstein, Imagenet large scale visual recognition challenge. International journal of computer vision, 2015. 115(3): p. 211-252.

43. Jin, X. and I.H. Riedel-Kruse, Biofilm Lithography enables high-resolution cell patterning via optogenetic adhesin expression. Proceedings of the National Academy of Sciences, 2018. 115(14): p. 3698-3703.

44. Chen, A.Y., Z. Deng, A.N. Billings, U.O.S. Seker, Michelle Y. Lu, R.J. Citorik, B. Zakeri, and T.K. Lu, Synthesis and patterning of tunable multiscale materials with engineered cells. Nature Materials, 2014. 13: p. 515.

45. Huang, J., S. Liu, C. Zhang, X. Wang, J. Pu, F. Ba, S. Xue, H. Ye, T. Zhao, K. Li, Y. Wang, J. Zhang, L. Wang, C. Fan, T.K. Lu, and C. Zhong, Programmable and printable Bacillus subtilis biofilms as engineered living materials. Nature Chemical Biology, 2019. 15(1): p. 34-41.

46. Luo, N., S. Wang, and L. You, Synthetic pattern formation. Biochemistry, 2019. 58(11): p. 1478-1483.

47. Nasip, Ö.F. and K. Zengin. Deep Learning Based Bacteria Classification. in 2018 2nd International Symposium on Multidisciplinary Studies and Innovative Technologies (ISMSIT). 2018. IEEE.

48. Casado-García, Á., G. Chichón, C. Domínguez, M. García-Domínguez, J. Heras, A. Inés, M. López, E. Mata, V. Pascual, and Y. Sáenz, MotilityJ: An open-source tool for the classification and segmentation of bacteria on motility images. Comput Biol Med, 2021. 136: p. 104673.

49. Wang, H., H.C. Koydemir, Y. Qu, B. Bai, Y. Zhang, Y. Jin, S. Tok, E.C. Yilmaz, E. Gumustekin, and Y. Rivenson, Early-detection and classification of live bacteria using time-lapse coherent imaging and deep learning. arXiv preprint arXiv:2001.10695, 2020.

50. Jeckel, H., E. Jelli, R. Hartmann, P.K. Singh, R. Mok, J.F. Totz, L. Vidakovic, B. Eckhardt, J. Dunkel, and K. Drescher, Learning the space-time phase diagram of bacterial swarm expansion. Proceedings of the National Academy of Sciences, 2019. 116(5): p. 1489-1494.

51. Lugagne, J.-B., H. Lin, and M.J. Dunlop, DeLTA: Automated cell segmentation, tracking, and lineage reconstruction using deep learning. PLoS computational biology, 2020. 16(4): p. e1007673.

52. Doshi, A., M. Shaw, R. Tonea, R. Minyety, S. Moon, A. Laine, J. Guo, and T. Danino, A deep learning pipeline for segmentation of $<e m>$ Proteus mirabilis $</$ em $>$ colony patterns. bioRxiv, 2022: p. 2022.01.17.475672.

53. Dietrich, L.E., T.K. Teal, A. Price-Whelan, and D.K. Newman, Redox-active antibiotics control gene expression and community behavior in divergent bacteria. Science, 2008. 321(5893): p. 1203-1206.

54. Minogue, T., H. Daligault, K. Davenport, K. Bishop-Lilly, D. Bruce, P. Chain, S. Coyne, O. Chertkov, T. Freitas, and K. Frey, Draft genome assemblies of Proteus mirabilis ATCC 7002 and Proteus vulgaris ATCC 49132. Genome announcements, 2014. 2(5). 
738

739

740

741

742

743

744

745

746

747

748

749

750

751

752

753

754

755

756

757

758

759

760

761

762

763

764

765

766

767

768

769

770

771

772

773

774

775
55. Pearson, M.M., Methods for Studying Swarming and Swimming Motility, in Proteus mirabilis: Methods and Protocols, M.M. Pearson, Editor. 2019, Springer New York: New York, NY. p. 15-25.

56. Tol, P., Colour Schemes. 2021, SRON: SRON/EPS/TN/09-002.

57. Kutschera, A. lightM. Available from: https://github.com/vektorious/lightM.

58. Levin-Reisman, I., O. Gefen, O. Fridman, I. Ronin, D. Shwa, H. Sheftel, and N.Q. Balaban, Automated imaging with ScanLag reveals previously undetectable bacterial growth phenotypes. Nature Methods, 2010. 7(9): p. 737-739.

59. Bär, J., M. Boumasmoud, R.D. Kouyos, A.S. Zinkernagel, and C. Vulin, Efficient microbial colony growth dynamics quantification with ColTapp, an automated image analysis application. Scientific reports, 2020. 10(1): p. 1-15.

60. Hartmann, R., H. Jeckel, E. Jelli, P.K. Singh, S. Vaidya, M. Bayer, D.K. Rode, L. Vidakovic, F. Díaz-Pascual, and J.C. Fong, Quantitative image analysis of microbial communities with BiofilmQ. Nature microbiology, 2021. 6(2): p. 151-156.

61. Pavlov, L. moving_polyfit. 2021 August 30, 2021]; Available from: https://www.mathworks.com/matlabcentral/fileexchange/86503-moving polyfit.

62. Hand, D.J. and R.J. Till, $A$ Simple Generalisation of the Area Under the ROC Curve for Multiple Class Classification Problems. Machine Learning, 2001. 45(2): p. 171-186.

63. Russell, B.C., A. Torralba, K.P. Murphy, and W.T. Freeman, LabelMe: a database and web-based tool for image annotation. International journal of computer vision, 2008. 77(1-3): p. 157-173.

64. Sundararajan, M., A. Taly, and Q. Yan. Axiomatic attribution for deep networks. in International Conference on Machine Learning. 2017. PMLR.

65. He, K., X. Zhang, S. Ren, and J. Sun, Deep Residual Learning for Image Recognition. 2016 IEEE Conference on Computer Vision and Pattern Recognition (CVPR), 2016: p. 770-778.

66. Szegedy, C., W. Liu, Y. Jia, P. Sermanet, S.E. Reed, D. Anguelov, D. Erhan, V. Vanhoucke, and A. Rabinovich, Going deeper with convolutions. 2015 IEEE Conference on Computer Vision and Pattern Recognition (CVPR), 2015: p. 1-9.

67. Szegedy, C., V. Vanhoucke, S. loffe, J. Shlens, and Z. Wojna, Rethinking the Inception Architecture for Computer Vision. 2016 IEEE Conference on Computer Vision and Pattern Recognition (CVPR), 2016: p. 2818-2826.

68. Ronneberger, O., P. Fischer, and T. Brox. U-Net: Convolutional Networks for Biomedical Image Segmentation. 2015. Cham: Springer International Publishing.

69. Iglovikov, V. and A. Shvets, Ternausnet: U-net with vgg11 encoder pre-trained on imagenet for image segmentation. arXiv preprint arXiv:1801.05746, 2018.

70. Simonyan, K. and A. Zisserman, Very deep convolutional networks for large-scale image recognition. arXiv preprint arXiv:1409.1556, 2014. 